\title{
ON A 2D VECTOR POISSON PROBLEM WITH APPARENTLY MUTUALLY EXCLUSIVE SCALAR BOUNDARY CONDITIONS
}

\author{
JeAn-Luc Guermond ${ }^{1}$, Luigi Quartapelle ${ }^{2}$ And Jiang Zhu ${ }^{3}$
}

\begin{abstract}
This work is devoted to the study of a two-dimensional vector Poisson equation with the normal component of the unknown and the value of the divergence of the unknown prescribed simultaneously on the entire boundary. These two scalar boundary conditions appear prima facie alternative in a standard variational framework. An original variational formulation of this boundary value problem is proposed here. Furthermore, an uncoupled solution algorithm is introduced together with its finite element approximation. The numerical scheme has been implemented and applied to solve a simple test problem.
\end{abstract}

Mathematics Subject Classification. 35J20, 35Q, 65N30, 76.

Received: July 31, 1998.

\section{INTRODUCTION}

The Poisson equation for a vector unknown in a bounded domain in two or three dimensions can be supplemented by different kinds of boundary conditions. Beside the simplest case of a vector Dirichlet condition leading to the uncoupled solution of independent scalar Dirichlet problems for the Cartesian components of the unknown, different combinations of Dirichlet and Neumann-like (i.e. derivative) boundary conditions can be specified on the boundary or on its parts. For instance, one can prescribe the tangential component(s) of the unknown on the entire boundary simultaneously with the value of the divergence of the vector field (see e.g. $[3,5,9])$; alternatively, one can impose the normal component together with the tangential component(s) of the curl of the vector unknown (see e.g. [9]). In both cases, these mixed (Dirichlet and derivative) boundary conditions are easily accommodated within the standard variational formulation of the vector elliptic problem obtained from the integration by parts and one arrives at coupled solution method for an operator endowed with the symmetry which is inherent to elliptic problems (see e.g. $[8,10-12,14-16]$ ).

There are, however, cases where the set of boundary conditions supplementing the vector Poisson equation does not fit within the usual variational formulation of the elliptic problem. For instance, in some 2D problems the normal component of the unknown is prescribed on the boundary simultaneously with the value of

\footnotetext{
Keywords and phrases. Vector Poisson equation, mutually exclusive scalar boundary conditions, variational formulation, uncoupled solution, finite element approximation.

1 Laboratoire d'Informatique pour la Mécanique et les Sciences de l'Ingénieur, CNRS, BP 133, 91403 Orsay, France.

e-mail: guermond@limsi.fr

2 Dipartimento di Fisica, Politecnico di Milano, Piazza Leonardo da Vinci, 32, 20133 Milano, Italy.

3 Laboratório Nacional de Computação Científica, MCT, Avenida Getúlio Vargas 333, Petrópolis, 25651-070 RJ, Brazil.

e-mail: jiang@lncc.br
} 
the divergence of the unknown and these two boundary conditions seem incompatible when one looks at the boundary integrals occurring in the variational formulation of the problem.

An even more serious difficulty is encountered in three dimensions when the two aforementioned scalar boundary conditions are to be satisfied simultaneously with a third scalar condition imposing the normal component of the curl of the vector unknown, a set of conditions found, for instance, to supplement the vector potential of the velocity field in incompressible flows [9]. In this case, beside the aforementioned apparent mutual incompatibility of the boundary conditions for the $2 \mathrm{D}$ problem, one has a derivative boundary condition for which there is no corresponding term at all in the surface integrals that could allow to enforce it as a natural boundary condition.

The aim of the present paper is to investigate the problem associated with a vector Poisson equation in two dimensions supplemented with the two boundary conditions imposing the values of the normal component and of the divergence, a combination of scalar boundary conditions which cannot be imposed according to existing variational formulations for vector elliptic problems. This investigation is to be considered as a preliminary step toward the study of the three-dimensional vector Poisson equation under the three scalar boundary conditions mentioned above.

The content of the paper is organized as follows. In Section 2 we give the preliminary definitions and introduce the variational formulation of an elliptic problem for a vector unknown subject to the two apparently mutually exclusive boundary conditions. The question of well-posedness of such a problem is addressed in Section 3, where the kernel of the linear operator associated with the problem is shown to be nontrivial, but only one-dimensional. Section 4 provides a similar analysis for the transposed operator. As a consequence of these results the variational problem we started from is modified and reformulated in a well-posed manner in Section 5. The interpretation of the modified variational problem as an elliptic boundary value problem is displayed in Section 6 which gives also the explicit expression of the compatibility condition for the problem with homogeneous boundary conditions. Section 7 extends the previous analysis to nonzero boundary data and includes the compatibility condition which the data of the problem must satisfy in the general case. In Section 8 we introduce a splitting method, which leads to an uncoupled numerical algorithm requiring to solve only scalar Poisson equations and an auxiliary problem for a scalar boundary unknown. The finite element approximation of the split solution algorithm is discussed in Section 9, while Section 10 details the error analysis of the uncoupled finite element method. The last section is devoted to a few numerical tests.

\section{Preliminaries AND Problem Definition}

Throughout this paper, we assume that $\Omega$ is an open and simply-connected domain of $\mathbb{R}^{2}$, with a Lipschitz continuous boundary $\Gamma$.

Definition 2.1. Hereafter we set

$$
\begin{gathered}
\boldsymbol{X}(\Omega)=\left\{\boldsymbol{v} \in \boldsymbol{L}^{2}(\Omega) \mid \boldsymbol{\nabla} \cdot \boldsymbol{v} \in L^{2}(\Omega), \nabla \times \boldsymbol{v} \in L^{2}(\Omega)\right\} \\
\boldsymbol{X}_{T}(\Omega)=\{\boldsymbol{v} \in \boldsymbol{X}(\Omega) \mid \boldsymbol{n} \cdot \boldsymbol{v}=0 \text { on } \Gamma\} \\
\boldsymbol{X}_{N}(\Omega)=\{\boldsymbol{v} \in \boldsymbol{X}(\Omega) \mid \boldsymbol{\tau} \cdot \boldsymbol{v}=0 \text { on } \Gamma\}
\end{gathered}
$$

We introduce the bilinear form

$$
a(\boldsymbol{u}, \boldsymbol{v})=(\nabla \times \boldsymbol{u}, \nabla \times \boldsymbol{v})+(\boldsymbol{\nabla} \cdot \boldsymbol{u}, \boldsymbol{\nabla} \cdot \boldsymbol{v}), \quad \forall \boldsymbol{u}, \boldsymbol{v} \in \boldsymbol{X}(\Omega)
$$


It is clear that $a \in \mathcal{L}(\boldsymbol{X}(\Omega) \times \boldsymbol{X}(\Omega) ; \mathbb{R})$ and $a$ is symmetric positive. Furthermore, it can be shown (see e.g. [6]) that the restriction of $a$ to the Hilbert spaces $\boldsymbol{X}_{T}(\Omega)$ and $\boldsymbol{X}_{N}(\Omega)$ induces a scalar product and that the associated norm is equivalent to the natural norm of $\boldsymbol{X}(\Omega)$. Hereafter we equip $\boldsymbol{X}_{T}(\Omega)$ and $\boldsymbol{X}_{N}(\Omega)$ with the following norm and scalar product:

$$
(\cdot, \cdot)_{1}=a(\cdot, \cdot) ; \quad|\cdot|_{1}=\{a(\cdot, \cdot)\}^{1 / 2} .
$$

The aim of the present paper is to analyze the following problem. Given $\boldsymbol{f} \in \boldsymbol{L}^{2}(\Omega)$

$$
\left\{\begin{array}{l}
\text { Find } \boldsymbol{u} \in \boldsymbol{X}_{T}(\Omega) \text { such that } \\
a(\boldsymbol{u}, \boldsymbol{v})=(\boldsymbol{f}, \boldsymbol{v}), \quad \forall \boldsymbol{v} \in \boldsymbol{X}_{N}(\Omega)
\end{array}\right.
$$

Remark 2.1. By standard arguments, it can be shown that problem (2.6) is formally equivalent to the following PDE's.

$$
\left\{\begin{aligned}
-\nabla^{2} \boldsymbol{u} & =\boldsymbol{f} & & \text { in } \Omega \\
\boldsymbol{\nabla} \cdot \boldsymbol{u} & =0 & & \text { on } \Gamma \\
\boldsymbol{n} \cdot \boldsymbol{u} & =0 & & \text { on } \Gamma
\end{aligned}\right.
$$

where the two boundary conditions seem a priori to be mutually exclusive.

The first question we have to answer is: Is problem (2.6) well-posed? An equivalent question consists in knowing whether the operator $A: \boldsymbol{X}_{T}(\Omega) \longrightarrow \boldsymbol{X}_{N}^{\prime}(\Omega)$ defined by

$$
\langle A \boldsymbol{u}, \boldsymbol{v}\rangle=a(\boldsymbol{u}, \boldsymbol{v}), \quad \forall \boldsymbol{u} \in \boldsymbol{X}_{T}(\Omega), \forall \boldsymbol{v} \in \boldsymbol{X}_{N}(\Omega),
$$

is bijective. The answer to this question is rooted in the study of the kernel of $A$ and that of $A^{t}$.

\section{Analysis of the Kernel of $A$}

Let us set

$$
L_{0}^{2}(\Omega)=\left\{q \in L^{2}(\Omega) \mid \int_{\Omega} g=0\right\}, \quad \text { and } \quad \widetilde{H}^{1}(\Omega)=H^{1}(\Omega) \cap L_{0}^{2}(\Omega) .
$$

Lemma 3.1. Let $g \in L_{0}^{2}(\Omega)$, then there exists a unique $\boldsymbol{v} \in \boldsymbol{X}_{N}(\Omega)$ such that

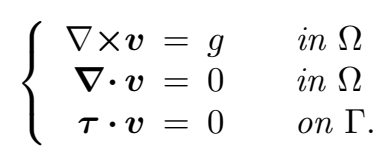

Proof. We show that the solution to (3.1) is indeed the solution to the following problem:

$$
\left\{\begin{array}{l}
\text { Find } \boldsymbol{v} \in \boldsymbol{X}_{N}(\Omega) \text { such that } \\
a(\boldsymbol{v}, \boldsymbol{w})=(g, \nabla \times \boldsymbol{w}), \quad \forall \boldsymbol{w} \in \boldsymbol{X}_{N}(\Omega) .
\end{array}\right.
$$

From the Riesz-Fréchet representation theorem we know that problem (3.2) has a unique solution. Let us prove now that $\boldsymbol{v}$ is solution to (3.1).

Next, for any $h \in L^{2}(\Omega)$, let $q \in H_{0}^{1}(\Omega)$ satisfying $\nabla^{2} q=h$ in $\Omega$, then $\boldsymbol{w}=\nabla q \in \boldsymbol{X}_{N}(\Omega)$. By (3.2) we have $(\boldsymbol{\nabla} \cdot \boldsymbol{v}, \boldsymbol{\nabla} \cdot \boldsymbol{w})=0$, i.e., $(\boldsymbol{\nabla} \cdot \boldsymbol{v}, \boldsymbol{\nabla} \cdot \boldsymbol{\nabla} q)=0$, namely,

$$
(\nabla \cdot \boldsymbol{v}, h)=0, \quad \forall h \in L^{2}(\Omega)
$$


which implies that $\boldsymbol{\nabla} \cdot \boldsymbol{v}=0$.

Finally, for any $\phi \in L_{0}^{2}(\Omega)$, let $\psi \in \widetilde{H}^{1}(\Omega)$ be the solution of

$$
\left\{\begin{array}{rlrl}
\nabla^{2} \psi & =\phi & & \text { in } \Omega \\
\frac{\partial \psi}{\partial n}=0 & & \text { on } \Gamma,
\end{array}\right.
$$

then $\boldsymbol{w}=\boldsymbol{\nabla} \times \psi \in \boldsymbol{X}_{N}(\Omega)$. By using $\boldsymbol{w}$ to test the equation of problem (3.2), we obtain:

$$
(\nabla \times \boldsymbol{v}, \nabla \times \nabla \times \psi)=(g, \nabla \times \nabla \times \psi),
$$

that is

$$
(\nabla \times \boldsymbol{v}-g, \phi)=0, \quad \forall \phi \in L_{0}^{2}(\Omega) .
$$

Thus, there exists a constant $C$ such that $\nabla \times \boldsymbol{v}-g=C$. Noticing that $\int_{\Omega} g=0$ and $\int_{\Omega} \nabla \times \boldsymbol{v}=\int_{\Gamma} \boldsymbol{\tau} \cdot \boldsymbol{v}=0$, we get $\nabla \times \boldsymbol{v}=g$. Finally, note that the solution of (3.1) is unique in $\boldsymbol{X}_{N}(\Omega)$.

We are now ready to study the kernel of the operator $A$.

Lemma 3.2. Let $\boldsymbol{u}_{0} \in \boldsymbol{X}(\Omega)$ be the solution of

$$
\left\{\begin{array}{rlrl}
\nabla \times \boldsymbol{u}_{0} & =1 & & \text { in } \Omega \\
\boldsymbol{\nabla} \cdot \boldsymbol{u}_{0}=0 & & \in \Omega \\
\boldsymbol{n} \cdot \boldsymbol{u}_{0}=0 & & \text { on } G .
\end{array}\right.
$$

Then, we have

$$
\text { Ker } A=\left\langle\boldsymbol{u}_{0}\right\rangle \text {. }
$$

Proof. Let $\boldsymbol{u} \in \boldsymbol{X}_{T}(\Omega)$ be in $\operatorname{Ker} A$. By definition, $\boldsymbol{u}$ satisfies

$$
a(\boldsymbol{u}, \boldsymbol{v})=0, \quad \forall \boldsymbol{v} \in \boldsymbol{X}_{N}(\Omega) .
$$

For any $g \in L_{0}^{2}(\Omega)$, by Lemma 3.1 we can find a $\boldsymbol{v} \in \boldsymbol{X}_{N}(\Omega)$ such that (3.1) holds. Thus, from (3.5) we have

$$
(\nabla \times \boldsymbol{u}, g)=0, \quad \forall g \in L_{0}^{2}(\Omega) .
$$

This implies that there is a constant $C_{u}$ such that

$$
\nabla \times \boldsymbol{u}=C_{u}
$$

Integrating this relation over $\Omega$ and using Stokes theorem we obtain

$$
C_{u}=\frac{1}{|\Omega|} \int_{\Gamma} \boldsymbol{\tau} \cdot \boldsymbol{u}
$$

On the other hand, for any $h \in L^{2}(\Omega)$, let $q \in H_{0}^{1}(\Omega)$ satisfying $\nabla^{2} q=h$ in $\Omega$. Then, by choosing $\boldsymbol{v}=\nabla q \in$ $\boldsymbol{X}_{N}(\Omega)$ in (3.5), we have $(\boldsymbol{\nabla} \cdot \boldsymbol{u}, \boldsymbol{\nabla} \cdot(\boldsymbol{\nabla} q))=0$, namely,

$$
(\boldsymbol{\nabla} \cdot \boldsymbol{u}, h)=0, \quad \forall h \in L^{2}(\Omega),
$$


which implies that

$$
\nabla \cdot u=0
$$

Summarizing the analysis above, we obtain that, if $\boldsymbol{u} \in \boldsymbol{X}_{T}(\Omega)$ is a solution of problem (3.5), then it satisfies the system

$$
\left\{\begin{aligned}
\nabla \times \boldsymbol{u} & =C_{u} \\
\nabla \cdot \boldsymbol{u} & =0 .
\end{aligned}\right.
$$

The converse is also true.

\section{Analysis of the transposed operator $A^{t}$}

We turn now to the study of the transpose of $A, A^{t}: \boldsymbol{X}_{N}(\Omega) \longrightarrow\left(\boldsymbol{X}_{T}(\Omega)\right)^{\prime}$, which is defined by

$$
\left\langle A^{t} \boldsymbol{v}, \boldsymbol{u}\right\rangle=a(\boldsymbol{u}, \boldsymbol{v}), \quad \forall \boldsymbol{u} \in \boldsymbol{X}_{T}(\Omega), \forall \boldsymbol{v} \in \boldsymbol{X}_{N}(\Omega) .
$$

Lemma 4.1. Let $\boldsymbol{v}_{0} \in \boldsymbol{X}(\Omega)$ be the solution of

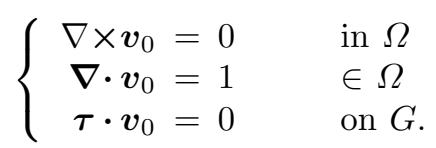

Then,

$$
\operatorname{Ker} A^{t}=\left\langle\boldsymbol{v}_{0}\right\rangle
$$

Proof. Let $\boldsymbol{v} \in \boldsymbol{X}_{N}(\Omega)$ satisfying

$$
a(\boldsymbol{u}, \boldsymbol{v})=0, \quad \forall \boldsymbol{u} \in \boldsymbol{X}_{T}(\Omega)
$$

For any $h \in L_{0}^{2}(\Omega)$, let $q \in \widetilde{H}^{1}(\Omega)$ be the solution of

$$
\left\{\begin{array}{rlrl}
\nabla^{2} q & =h & & \text { in } \Omega \\
\frac{\partial q}{\partial n}=0 & & \text { on } \Gamma .
\end{array}\right.
$$

Then, by taking $\boldsymbol{u}=\boldsymbol{\nabla} q \in \boldsymbol{X}_{T}(\Omega)$ in (4.4), we have $(\boldsymbol{\nabla} \cdot(\boldsymbol{\nabla} q), \boldsymbol{\nabla} \cdot \boldsymbol{v})=0$, that is

$$
(\nabla \cdot \boldsymbol{v}, h)=0, \quad \forall h \in L_{0}^{2}(\Omega),
$$

which implies that

$$
\nabla \cdot \boldsymbol{v}=C_{v}
$$

where

$$
C_{v}=\frac{1}{|\Omega|} \int_{\Gamma} \boldsymbol{n} \cdot \boldsymbol{v}
$$


On the other hand, for any $g \in L^{2}(\Omega)$, let $\phi \in H_{0}^{1}(\Omega)$ be such that $\nabla^{2} \phi=g$ in $\Omega$. Thus, $\boldsymbol{u}=\boldsymbol{\nabla} \times \phi \in \boldsymbol{X}_{T}(\Omega)$ and (4.4) becomes $(\nabla \times \nabla \times \phi, \nabla \times \boldsymbol{v})=0$, i.e.,

$$
(\nabla \times \boldsymbol{v}, g)=0, \quad \forall g \in L^{2}(\Omega),
$$

which means that

$$
\nabla \times v=0
$$

Consequently, we obtain that, if $\boldsymbol{v} \in \boldsymbol{X}_{N}(\Omega)$ is a solution of problem (4.4), then it satisfies the system

$$
\left\{\begin{aligned}
\nabla \times \boldsymbol{v} & =0 \\
\boldsymbol{\nabla} \cdot \boldsymbol{v} & =C_{\boldsymbol{v}} .
\end{aligned}\right.
$$

The converse is also true.

\section{A WELL-POSED PROBLEM}

The above analysis has shown that problem (2.6) is not well-posed, for a necessary condition for an operator to be bijective is that the operator and its transpose are injective. In order to exclude $\left\langle\boldsymbol{u}_{0}\right\rangle$ and $\left\langle\boldsymbol{v}_{0}\right\rangle$ from $\boldsymbol{X}_{T}(\Omega)$ and $\boldsymbol{X}_{N}(\Omega)$ respectively, we set

\section{Definition 5.1.}

$$
\begin{aligned}
& \boldsymbol{X}_{T}^{\star}(\Omega)=\left\{\boldsymbol{v} \in \boldsymbol{X}_{T}(\Omega) \mid \int_{\Gamma} \boldsymbol{\tau} \cdot \boldsymbol{v}=0\right\} \\
& \boldsymbol{X}_{N}^{\star}(\Omega)=\left\{\boldsymbol{v} \in \boldsymbol{X}_{N}(\Omega) \mid \int_{\Gamma} \boldsymbol{n} \cdot \boldsymbol{v}=0\right\} .
\end{aligned}
$$

Instead of problem (2.6), we shall hereafter consider the following one

$$
\left\{\begin{array}{l}
\text { Find } \boldsymbol{u} \in \boldsymbol{X}_{T}^{\star}(\Omega) \text { such that } \\
a(\boldsymbol{u}, \boldsymbol{v})=(\boldsymbol{f}, \boldsymbol{v}), \quad \forall \boldsymbol{v} \in \boldsymbol{X}_{N}^{\star}(\Omega) .
\end{array}\right.
$$

Theorem 5.1. Problem (5.3) is well-posed.

Proof. According to the global theory on linear variational problems (cf. [2]), problem (5.3) is well-posed if and only if the following two conditions are satisfied:

(i) there exists a constant $\alpha>0$ such that

$$
\inf _{\boldsymbol{u} \in \boldsymbol{X}_{T}^{\star}(\Omega) \backslash\{\mathbf{0}\}} \sup _{\boldsymbol{v} \in \boldsymbol{X}_{N}^{\star}(\Omega) \backslash\{\mathbf{0}\}} \frac{a(\boldsymbol{u}, \boldsymbol{v})}{|\boldsymbol{u}|_{1}|\boldsymbol{v}|_{1}} \geq \alpha,
$$

(ii) for any $\boldsymbol{v} \neq \mathbf{0}$ in $\boldsymbol{X}_{N}^{\star}(\Omega)$

$$
\sup _{\boldsymbol{u} \in \boldsymbol{X}_{T}^{\star}(\Omega)} a(\boldsymbol{u}, \boldsymbol{v})>0 .
$$


Given $\boldsymbol{u} \in \boldsymbol{X}_{T}^{\star}(\Omega)$, let us consider the following problem

$$
\left\{\begin{array}{l}
\text { Find } \boldsymbol{w} \in \boldsymbol{X}_{N}^{\star}(\Omega) \text { such that } \\
a\left(\boldsymbol{w}, \boldsymbol{v}^{\prime}\right)=a\left(\boldsymbol{u}, \boldsymbol{v}^{\prime}\right), \quad \forall \boldsymbol{v}^{\prime} \in \boldsymbol{X}_{N}^{\star}(\Omega) .
\end{array}\right.
$$

By the Riesz-Fréchet Theorem, problem (5.6) has a unique solution $\boldsymbol{w} \in \boldsymbol{X}_{N}^{\star}(\Omega)$, and

$$
|\boldsymbol{w}|_{1} \leq|\boldsymbol{u}|_{1}
$$

We are going to show that $\boldsymbol{w}$ satisfies

$$
\left\{\begin{aligned}
\nabla \times \boldsymbol{w} & =\nabla \times \boldsymbol{u} & & \text { in } \Omega \\
\nabla \cdot \boldsymbol{w} & =\nabla \cdot \boldsymbol{u} & & \text { in } \Omega .
\end{aligned}\right.
$$

For any $h \in L_{0}^{2}(\Omega)$, let $q \in \widetilde{H}^{1}(\Omega)$ be the solution of

$$
\left\{\begin{array}{rlrl}
\nabla^{2} q & =h & & \text { in } \Omega \\
\frac{\partial q}{\partial n}=0 & & \text { on } \Gamma
\end{array}\right.
$$

Then $\boldsymbol{v}^{\prime}=\nabla \times q \in \boldsymbol{X}_{N}^{\star}(\Omega)$. By (5.6), we have

$$
(\nabla \times \boldsymbol{w}, h)=(\nabla \times \boldsymbol{u}, h), \quad \forall h \in L_{0}^{2}(\Omega),
$$

which implies that the first relation of (5.8) holds, since $\boldsymbol{w} \in \boldsymbol{X}_{N}^{\star}(\Omega)$ and $\boldsymbol{u} \in \boldsymbol{X}_{T}^{\star}(\Omega)$.

Next, for any $h \in L_{0}^{2}(\Omega)$, let $\phi \in H_{0}^{1}(\Omega)$ satisfying $\nabla^{2} \phi=h$ in $\Omega$, then $\boldsymbol{v}^{\prime}=\nabla \phi \in \boldsymbol{X}_{N}^{\star}(\Omega)$. From (5.6), we have

$$
(\boldsymbol{\nabla} \cdot \boldsymbol{w}, h)=(\boldsymbol{\nabla} \cdot \boldsymbol{u}, h), \quad \forall h \in L_{0}^{2}(\Omega)
$$

Since $\boldsymbol{w} \in \boldsymbol{X}_{N}^{\star}(\Omega)$ and $\boldsymbol{u} \in \boldsymbol{X}_{T}^{\star}(\Omega),(5.9)$ implies that the second relation of (5.8) holds.

Let us now check conditions (5.4) and (5.5). Notice that (5.8), we have

$$
\sup _{\boldsymbol{v} \in \boldsymbol{X}_{N}^{\star}(\Omega) \backslash\{\boldsymbol{0}\}} \frac{a(\boldsymbol{u}, \boldsymbol{v})}{|\boldsymbol{u}|_{1}|\boldsymbol{v}|_{1}} \geq \frac{a(\boldsymbol{u}, \boldsymbol{w})}{|\boldsymbol{u}|_{1}|\boldsymbol{w}|_{1}}=1
$$

i.e. condition (5.4) is satisfied.

To establish (5.5), let us assume that a given nonzero $\boldsymbol{v} \in \boldsymbol{X}_{N}^{\star}(\Omega)$ satisfies:

$$
a(\boldsymbol{u}, \boldsymbol{v})=0, \quad \forall \boldsymbol{u} \in \boldsymbol{X}_{T}^{\star}(\Omega) .
$$

Similarly to the arguments used to prove (4.8), we can get

$$
\begin{cases}\nabla \times \boldsymbol{v}=0 & \text { in } \Omega \\ \nabla \cdot \boldsymbol{v}=0 & \text { in } \Omega\end{cases}
$$

Thus, $\boldsymbol{v}$ should be zero, which leads to a contradiction. Therefore, condition (5.5) is also satisfied. This completes the proof. 
Remark 5.1. Theorem 5.1 is equivalent to stating that the operator $A^{\star}: \boldsymbol{X}_{T}^{\star}(\Omega) \longrightarrow\left(\boldsymbol{X}_{N}^{\star}(\Omega)\right)^{\prime}$ defined by

$$
\left\langle A^{\star} \boldsymbol{u}, \boldsymbol{v}\right\rangle=a(\boldsymbol{u}, \boldsymbol{v}), \quad \forall \boldsymbol{u} \in \boldsymbol{X}_{T}^{\star}(\Omega), \forall \boldsymbol{v} \in \boldsymbol{X}_{N}^{\star}(\Omega),
$$

is bijective.

\section{INTERPRETATION OF THE WELL-POSED PROBLEM}

To interpret the well-posed problem (5.3) in a strong form, we have:

Theorem 6.1. If $\int_{\Omega} \boldsymbol{f} \cdot \boldsymbol{v}_{0}=0$ where $\boldsymbol{v}_{0}$ is defined by (4.2), then the solution $\boldsymbol{u}$ of problem (5.3) satisfies:

$$
\left\{\begin{aligned}
-\nabla^{2} \boldsymbol{u} & =\boldsymbol{f} & & \text { in } \Omega \\
\boldsymbol{\nabla} \cdot \boldsymbol{u} & =0 & & \text { on } \Gamma \\
\boldsymbol{n} \cdot \boldsymbol{u} & =0 & & \text { on } \Gamma \\
\int_{\Gamma} \boldsymbol{\tau} \cdot \boldsymbol{u} & =0 . & &
\end{aligned}\right.
$$

Proof. By the definition of $\boldsymbol{v}_{0}$ and the assumption,

$$
a\left(\boldsymbol{u}, \boldsymbol{v}_{0}\right)=0=\left(\boldsymbol{f}, \boldsymbol{v}_{0}\right)
$$

Noticing that

$$
\boldsymbol{X}_{N}(\Omega)=\boldsymbol{X}_{N}^{\star}(\Omega) \oplus\left\langle\boldsymbol{v}_{0}\right\rangle
$$

we infer that the solution $\boldsymbol{u}$ of problem (5.3) satisfies

$$
a(\boldsymbol{u}, \boldsymbol{v})=(\boldsymbol{f}, \boldsymbol{v}), \quad \forall \boldsymbol{v} \in \boldsymbol{X}_{N}(\Omega),
$$

which is equivalent to (6.1) by virtue of Remark 2.1.

Remark 6.1. Let $\kappa_{0}$ be such that

$$
\left\{\begin{aligned}
\nabla^{2} \kappa_{0}=1 & \text { in } \Omega \\
\kappa_{0}=0 & \text { on } \Gamma .
\end{aligned}\right.
$$

Then, $\boldsymbol{v}_{0}=\nabla \kappa_{0}$ and the assumption of Theorem 6.1 can be expressed in the form

$$
\int_{\Omega} f \cdot \nabla \kappa_{0}=0
$$

which is the compatibility condition of problem (6.1).

\section{NONHOMOGENEOUS BOUNDARY CONDITIONS}

We now consider the case of nonhomogeneous boundary conditions, that is the following boundary value problem:

$$
\left\{\begin{aligned}
-\nabla^{2} \boldsymbol{u} & =\boldsymbol{f} & & \text { in } \Omega \\
\boldsymbol{\nabla} \cdot \boldsymbol{u} & =d & & \text { on } \Gamma \\
\boldsymbol{n} \cdot \boldsymbol{u} & =b & & \text { on } \Gamma \\
\int_{\Gamma}^{\boldsymbol{\tau} \cdot \boldsymbol{u}} & =e & &
\end{aligned}\right.
$$


where $\boldsymbol{f} \in \boldsymbol{L}^{2}(\Omega), d \in H^{1 / 2}(\Gamma), b \in H^{-1 / 2}(\Gamma)$ and $e \in \mathbb{R}$.

To solve problem (7.1), one has to show how its boundary conditions and its integral constraint can be homogeneized. To this purpose, let $p \in \widetilde{H}^{1}(\Omega)$ be the solution of the Neumann problem

$$
\left\{\begin{aligned}
\nabla^{2} p & =\frac{\bar{b}}{|\Omega|} & & \text { in } L^{2}(\Omega) \\
\frac{\partial p}{\partial n} & =b & & \text { in } H^{-1 / 2}(\Gamma)
\end{aligned}\right.
$$

where

$$
\bar{b}=\int_{\Gamma} b,
$$

and $H^{-1 / 2}(\Gamma) \subset L^{1}(\Gamma)$ guarantees that problem (7.3) is well defined. Then $\boldsymbol{u}_{b}=\nabla p$ satisfies:

$$
\left\{\begin{array}{rlrl}
\nabla \cdot \boldsymbol{u}_{b} & \in L^{2}(\Omega) & \\
\nabla \times \boldsymbol{u}_{b} & \in L^{2}(\Omega) & & \\
\nabla^{2} \boldsymbol{u}_{b} & =\mathbf{0} & & \text { in } \Omega \\
\boldsymbol{\nabla} \cdot \boldsymbol{u}_{b} & =\frac{\bar{b}}{|\Omega|} & & \text { on } \Gamma \\
\boldsymbol{n} \cdot \boldsymbol{u}_{b} & =b & & \text { on } \Gamma \\
\int_{\Gamma} \boldsymbol{\tau} \cdot \boldsymbol{u}_{b} & =0 . & &
\end{array}\right.
$$

On the other hand, we define

$$
\boldsymbol{u}_{e}=-\frac{e}{|\Omega|} \nabla \times \kappa_{0}
$$

where $\kappa_{0}$ is defined by $(6.3)$. It is easy to check that

$$
\left\{\begin{aligned}
\nabla \cdot \boldsymbol{u}_{e} & \in L^{2}(\Omega) & & \\
\nabla \times \boldsymbol{u}_{e} & \in L^{2}(\Omega) & & \\
\nabla^{2} \boldsymbol{u}_{e} & =\mathbf{0} & & \text { in } \Omega \\
\boldsymbol{\nabla} \cdot \boldsymbol{u}_{e} & =0 & & \text { on } \Gamma \\
\boldsymbol{n} \cdot \boldsymbol{u}_{e} & =0 & & \text { on } \Gamma \\
\int_{\Gamma} \boldsymbol{\tau} \cdot \boldsymbol{u}_{e} & =e & &
\end{aligned}\right.
$$

Therefore,

$$
\left\{\begin{aligned}
-\nabla^{2}\left(\boldsymbol{u}-\boldsymbol{u}_{b}-\boldsymbol{u}_{e}\right) & =\boldsymbol{f} & & \text { in } \Omega \\
\nabla \cdot\left(\boldsymbol{u}-\boldsymbol{u}_{b}-\boldsymbol{u}_{e}\right) & =d-\frac{\bar{b}}{|\Omega|} & & \text { on } \Gamma \\
\boldsymbol{n} \cdot\left(\boldsymbol{u}-\boldsymbol{u}_{b}-\boldsymbol{u}_{e}\right) & =0 & & \text { on } \Gamma \\
\int_{\Gamma} \boldsymbol{\tau} \cdot\left(\boldsymbol{u}-\boldsymbol{u}_{b}-\boldsymbol{u}_{e}\right) & =0 . & &
\end{aligned}\right.
$$


To solve problem (7.7), let us consider the following problem

$$
\left\{\begin{array}{l}
\text { Find } \boldsymbol{\phi} \in \boldsymbol{X}_{T}^{\star}(\Omega) \text { such that } \\
a(\boldsymbol{\phi}, \boldsymbol{v})=(\boldsymbol{f}, \boldsymbol{v})+\int_{\Gamma}\left(d-\frac{\bar{b}}{|\Omega|}\right)(\boldsymbol{n} \cdot \boldsymbol{v}), \quad \forall \boldsymbol{v} \in \boldsymbol{X}_{N}^{\star}(\Omega) .
\end{array}\right.
$$

Since

$$
\begin{aligned}
\left|\int_{\Gamma}\left(d-\frac{\bar{b}}{|\Omega|}\right)(\boldsymbol{n} \cdot \boldsymbol{v})\right| & \leq\left|d-\frac{\bar{b}}{|\Omega|}\right|_{1 / 2, \Gamma}|\boldsymbol{n} \cdot \boldsymbol{v}|_{-1 / 2, \Gamma} \\
& \leq\left|d-\frac{\bar{b}}{|\Omega|}\right|_{1 / 2, \Gamma}|\boldsymbol{v}|_{\boldsymbol{H}(\operatorname{div}, \Omega)} \\
& \leq\left|d-\frac{\bar{b}}{|\Omega|}\right|_{1 / 2, \Gamma}|\boldsymbol{v}|_{1},
\end{aligned}
$$

the linear form $\boldsymbol{v}: \longrightarrow(\boldsymbol{f}, \boldsymbol{v})+\int_{\Gamma}\left(d-\frac{\bar{b}}{|\Omega|}\right)(\boldsymbol{n} \cdot \boldsymbol{v})$ is continuous on $\boldsymbol{X}_{N}^{\star}(\Omega)$. So, problem (7.8) is well-posed.

We assume that

$$
\left(\boldsymbol{f}, \boldsymbol{v}_{0}\right)+\int_{\Gamma}\left(d-\frac{\bar{b}}{|\Omega|}\right)\left(\boldsymbol{n} \cdot \boldsymbol{v}_{0}\right)=0,
$$

where $\boldsymbol{v}_{0}=\boldsymbol{\nabla} \kappa_{0}$. Since $\int_{\Gamma} \boldsymbol{n} \cdot \boldsymbol{v}_{0}=\int_{\Omega} \nabla^{2} \kappa_{0}=|\Omega|$, the above assumption can be written as

$$
\left(\boldsymbol{f}, \nabla \kappa_{0}\right)-\bar{b}+\int_{\Gamma} d \frac{\partial \kappa_{0}}{\partial n}=0
$$

Under this hypothesis, the solution $\phi$ of problem (7.8) satisfies also

$$
a(\boldsymbol{\phi}, \boldsymbol{v})=(\boldsymbol{f}, \boldsymbol{v})+\int_{\Gamma}\left(d-\frac{\bar{b}}{|\Omega|}\right)(\boldsymbol{n} \cdot \boldsymbol{v}), \quad \forall \boldsymbol{v} \in \boldsymbol{X}_{N}(\Omega) .
$$

If we introduce the space:

$$
\boldsymbol{X}_{b, e}(\Omega)=\left\{\boldsymbol{v} \in \boldsymbol{X} \mid \boldsymbol{n} \cdot \boldsymbol{v}=b \text { on } \Gamma \text { and } \int_{\Gamma} \boldsymbol{\tau} \cdot \boldsymbol{v}=e\right\},
$$

then the variational form of problem (7.1) can be written as

$$
\left\{\begin{array}{l}
\text { Find } \boldsymbol{u} \in \boldsymbol{X}_{b, e}(\Omega) \text { such that } \\
a(\boldsymbol{u}, \boldsymbol{v})=(\boldsymbol{f}, \boldsymbol{v})+\langle d, \boldsymbol{n} \cdot \boldsymbol{v}\rangle_{1 / 2, \Gamma}, \quad \forall \boldsymbol{v} \in \boldsymbol{X}_{N}(\Omega) .
\end{array}\right.
$$

Therefore, we have:

Theorem 7.1. Assume that $\Omega$ is $C^{0,1}$ (i.e. Lipschitzian), $\boldsymbol{f} \in \boldsymbol{L}^{2}(\Omega), d \in H^{1 / 2}(\Gamma), b \in H^{-1 / 2}(\Gamma)$ and $e \in \mathbb{R}$. Then, under Hypothesis (H1), the variational problem (7.11) has a unique solution.

\section{A split SOlUtion METHOD AND its VARIATIONAL FORMUlation}

We can split problem (7.1) into a sequence of uncoupled simple problems. First, for the given data $\boldsymbol{f} \in \boldsymbol{L}^{2}(\Omega)$ and $d \in H^{1 / 2}(\Gamma)$, the following Dirichlet problem (to get $\phi=\boldsymbol{\nabla} \cdot \boldsymbol{u}$ )

$$
\left\{\begin{aligned}
-\nabla^{2} \phi & =\nabla \cdot f & & \text { in } \Omega \\
\phi & =d & & \text { on } \Gamma
\end{aligned}\right.
$$


has a unique solution $\phi \in H^{1}(\Omega)$. Notice that, by the definition of $\kappa_{0}$ and by Hypothesis (H1), we have

$$
\begin{aligned}
\int_{\Omega} \phi & =\int_{\Omega} \phi \nabla^{2} \kappa_{0} \\
& =\int_{\Omega}\left(\nabla^{2} \phi\right) \kappa_{0}+\int_{\Gamma} d \frac{\partial \kappa_{0}}{\partial n} \\
& =-\int_{\Omega}(\boldsymbol{\nabla} \cdot \boldsymbol{f}) \kappa_{0}+\int_{\Gamma} d \frac{\partial \kappa_{0}}{\partial n} \\
& =\int_{\Omega} \boldsymbol{f} \cdot \nabla \kappa_{0}+\int_{\Gamma} d \frac{\partial \kappa_{0}}{\partial n} \\
& =\bar{b} \\
& =\int_{\Gamma} b .
\end{aligned}
$$

This implies that the following Neumann problem

$$
\left\{\begin{array}{rlrl}
\nabla^{2} q & =\phi & & \text { in } \Omega \\
\frac{\partial q}{\partial n}=b & & \text { on } \Gamma
\end{array}\right.
$$

with $b \in H^{-1 / 2}(\Gamma) \subset L^{1}(\Gamma)$, has a unique solution $q \in \widetilde{H}^{1}(\Omega)$.

By the theory developed recently in $[14,15]$ or by $(8.11)$ (see below), we know that the following problem (which is the $2 \mathrm{D}$ version with homogeneous conditions of the boundary value problem introduced by Quartapelle and Muzzio [10])

$$
\left\{\begin{aligned}
-\nabla^{2} \boldsymbol{\psi} & =\boldsymbol{f}+\boldsymbol{\nabla} \phi & & \text { in } \Omega \\
\boldsymbol{\nabla} \cdot \boldsymbol{\psi} & =0 & & \text { on } \Gamma \\
\boldsymbol{\tau} \cdot \boldsymbol{\psi} & =0 & & \text { on } \Gamma
\end{aligned}\right.
$$

has a unique solution $\boldsymbol{\psi} \in \boldsymbol{X}_{N}(\Omega)$. As a consequence, the following scalar Dirichlet problem with homogeneous boundary condition

$$
\left\{\begin{aligned}
-\nabla^{2} w & =\nabla \times \psi & & \text { in } \Omega \\
w & =0 & & \text { on } \Gamma
\end{aligned}\right.
$$

has also a unique solution $w \in H^{1}(\Omega)$. Therefore, we can check that the vector field

$$
\boldsymbol{\nabla} \times\left(w-\frac{e}{|\Omega|} \kappa_{0}\right)+\nabla q=\boldsymbol{u} \in \boldsymbol{X}(\Omega)
$$

is the solution of problem (7.1).

Remark 8.1. The above splitting process provides another proof of Theorem 7.1. In fact, the existence is obtained by (8.5). For the uniqueness, we just need to consider the following homogeneous problem

$$
\left\{\begin{aligned}
\nabla^{2} \boldsymbol{v} & =\mathbf{0} & & \text { in } \Omega \\
\boldsymbol{n} \cdot \boldsymbol{v} & =0 & & \text { on } \Gamma \\
\boldsymbol{\nabla} \cdot \boldsymbol{v} & =0 & & \text { on } \Gamma \\
\int_{\Gamma} \boldsymbol{\tau} \cdot \boldsymbol{v} & =0 . & &
\end{aligned}\right.
$$


This problem is equivalent to

$$
\begin{cases}\nabla \times \boldsymbol{v}=0 & \text { in } \Omega \\ \boldsymbol{\nabla} \cdot \boldsymbol{v}=0 & \text { in } \Omega \\ \boldsymbol{n} \cdot \boldsymbol{v}=0 & \text { on } \Gamma .\end{cases}
$$

Obviously, problem (8.7) has a unique solution $\boldsymbol{v}=\mathbf{0}$, so does problem (8.6).

The solution of problem (7.1) can be determined by solving the sequence of problems form (8.1) to (8.5). Stated in variational form, all these problems read:

For

$$
\begin{aligned}
& H_{d}^{1}(\Omega)=\left\{\varphi \in H^{1}(\Omega) \mid \varphi=d \text { on } \Gamma\right\} \\
& \left\{\begin{array}{l}
\text { Find } \phi \in H_{d}^{1}(\Omega) \text { such that } \\
(\boldsymbol{\nabla} \phi, \nabla \varphi)=-(\boldsymbol{f}, \boldsymbol{\nabla} \varphi), \quad \forall \varphi \in H_{0}^{1}(\Omega) .
\end{array}\right. \\
& \left\{\begin{array}{l}
\text { Find } q \in \widetilde{H}^{1}(\Omega) \text { such that } \\
(\nabla q, \nabla \varphi)=-(\phi, \varphi)+\langle\varphi, b\rangle_{1 / 2, \Gamma}, \quad \forall \varphi \in \widetilde{H}^{1}(\Omega) .
\end{array}\right. \\
& \left\{\begin{array}{l}
\text { Find } \boldsymbol{\psi} \in \boldsymbol{X}_{N}(\Omega) \text { such that } \\
(\boldsymbol{\psi}, \boldsymbol{\varphi})_{1}=(\boldsymbol{f}+\nabla \phi, \boldsymbol{\varphi}), \quad \forall \boldsymbol{\varphi} \in \boldsymbol{X}_{N}(\Omega) .
\end{array}\right. \\
& \left\{\begin{array}{l}
\text { Find } w \in H_{0}^{1}(\Omega) \text { such that } \\
(\boldsymbol{\nabla} w, \boldsymbol{\nabla} \varphi)=(\boldsymbol{\psi}, \boldsymbol{\nabla} \times \varphi), \quad \forall \varphi \in H_{0}^{1}(\Omega) .
\end{array}\right. \\
& \left\{\begin{array}{l}
\text { Find } \boldsymbol{u} \in \boldsymbol{L}^{2}(\Omega) \text { such that } \\
(\boldsymbol{u}, \boldsymbol{\varphi})=(\boldsymbol{\nabla} \times w+\nabla q, \boldsymbol{\varphi}), \quad \forall \boldsymbol{\varphi} \in \boldsymbol{L}^{2}(\Omega) .
\end{array}\right.
\end{aligned}
$$

According to the results in $[14,15]$, the solution of the 2D homogeneous Quartapelle-Muzzio problem (8.11) can be split into

$$
\psi=\psi_{0}+\psi_{\mathcal{H}}
$$

where $\boldsymbol{\psi}_{0} \in \boldsymbol{H}_{0}^{1}(\Omega)$ and $\boldsymbol{\psi}_{\mathcal{H}} \in \mathcal{H}_{N}(\Omega)$, having introduced the following space of harmonic vector fields:

$$
\mathcal{H}_{N}(\Omega)=\left\{\boldsymbol{v} \in \boldsymbol{X}_{N}(\Omega) \mid(\boldsymbol{v}, \boldsymbol{w})_{1}=0, \forall \boldsymbol{w} \in \boldsymbol{H}_{0}^{1}(\Omega)\right\}
$$

Then, problem (8.11) can be written in the following form:

$$
\left\{\begin{aligned}
\text { Find }\left(\boldsymbol{\psi}_{0}, \boldsymbol{\psi}_{\mathcal{H}}\right) \in \boldsymbol{H}_{0}^{1}(\Omega) \times \mathcal{H}_{N}(\Omega) \text { such that } & \\
\text { (i) } \quad\left(\boldsymbol{\psi}_{0}, \boldsymbol{\varphi}_{0}\right)_{1}=\left(\boldsymbol{f}+\boldsymbol{\nabla} \phi, \boldsymbol{\varphi}_{0}\right), & \forall \boldsymbol{\varphi}_{0} \in \boldsymbol{H}_{0}^{1}(\Omega), \\
\text { (ii) }\left(\boldsymbol{\psi}_{\mathcal{H}}, \boldsymbol{\varphi}_{\mathcal{H}}\right)_{1}=-\left(\boldsymbol{\psi}_{0}, \boldsymbol{\varphi}_{\mathcal{H}}\right)_{1}+\left(\boldsymbol{f}+\boldsymbol{\nabla} \phi, \boldsymbol{\varphi}_{\mathcal{H}}\right), & \forall \boldsymbol{\varphi}_{\mathcal{H}} \in \mathcal{H}_{N}(\Omega) .
\end{aligned}\right.
$$


Subproblem (8.16.i) is a classical vector Dirichlet problem and is easily solved as two independent scalar Dirichlet problems. Subproblem (8.16.ii) is a vector problem whose solution cannot be found, in general, by solving two uncoupled scalar problems, since the space $\mathcal{H}_{N}(\Omega)$ brings about a coupling between the two vector components of the unknown $\boldsymbol{\psi}_{\mathcal{H}}$.

Summarizing the above results, we have:

Theorem 8.1. Problem (7.1) can be solved by the sequence of variational problems (8.9), (8.10), (8.16), (8.12) and (8.13).

\section{Finite element approximation}

Throughout this section, $\Omega$ is assumed to be a polygonal domain. Let $\mathcal{T}^{h}$ be a triangulation of $\bar{\Omega}$ such that $\boldsymbol{n}$ is a constant vector along $s \in \Sigma^{h}$, where $\Sigma^{h}$ is the set of segments contained in $\Gamma$, which are edges of an element of $\mathcal{T}^{h}$. Let $k \geq 1$ be an integer number. We introduce some finite element spaces:

$$
\begin{aligned}
S^{h, k} & =\left\{\varphi^{h} \in C^{0}(\bar{\Omega})\left|\varphi^{h}\right|_{\kappa} \in P_{k}, \forall \kappa \in \mathcal{T}^{h}\right\}, \\
S_{0}^{h, k} & =S^{h, k} \cap H_{0}^{1}(\Omega), \\
S_{d}^{h, k} & =\left\{\varphi^{h} \in S^{h, k}\left|\varphi^{h}\right|_{s}=\mathcal{I}_{k} d, \forall s \in \Sigma^{h}\right\}, \\
S_{n}^{h, k} & =\left\{\varphi^{h} \in S^{h, k} \mid \int_{\Omega} \varphi^{h}=0\right\},
\end{aligned}
$$

where $P_{k}$ denotes the space of all polynomials defined in $\mathbb{R}^{2}$, of degree less than or equal to $k \geq 1, \mathcal{I}_{k}$ denotes the standard Lagrange $P_{k}$-interpolation operator over $s$.

To approximate $\mathcal{H}_{N}(\Omega)$, as in [14-16], we introduce a space of discrete harmonic vector fields, defined by

$$
\mathcal{H}_{N}^{h, k}=\left\{\varphi^{h} \in \boldsymbol{S}_{N}^{h, k} \mid\left(\varphi^{h}, \boldsymbol{v}^{h}\right)_{1}=0, \forall \boldsymbol{v}^{h} \in\left[S_{0}^{h, k}\right]^{2}\right\}
$$

where

$$
\boldsymbol{S}_{N}^{h, k}=\left\{\boldsymbol{\varphi}^{h} \in\left[S^{h, k}\right]^{2}\left|\boldsymbol{\tau} \cdot \boldsymbol{\varphi}^{h}\right|_{s}=0, \forall s \in \Sigma^{h}\right\}
$$

According to the assumption on the triangulation $\mathcal{T}^{h}$, it is easy to see that each vector function of space $\boldsymbol{S}_{N}^{h, k}$, and therefore also of $\mathcal{H}_{N}^{h, k}$, can be written into two scalar functions of $S^{h, k}$.

Then, finite element approximations to the sequence of problems $(8.9,8.10,8.16,8.12)$ and $(8.13)$ can be proposed as follows: for $j=k$ or $k+1$,

$$
\begin{aligned}
& \left\{\begin{array}{l}
\text { Find } \phi^{h} \in S_{d}^{h, k} \text { such that } \\
\left(\boldsymbol{\nabla} \phi^{h}, \nabla \varphi^{h}\right)=-\left(\boldsymbol{f}, \nabla \varphi^{h}\right), \quad \forall \varphi^{h} \in S_{0}^{h, k},
\end{array}\right. \\
& \left\{\begin{array}{l}
\text { Find } q^{h} \in S_{n}^{h, j} \text { such that } \\
\left(\nabla q^{h}, \nabla \varphi^{h}\right)=-\left(\phi^{h}, \varphi^{h}\right)+\left\langle\varphi^{h}, b\right\rangle_{1 / 2, \Gamma}, \quad \forall \varphi^{h} \in S_{n}^{h, j},
\end{array}\right.
\end{aligned}
$$




$$
\begin{aligned}
& \left\{\begin{array}{l}
\text { Find } w^{h} \in S_{0}^{h, j} \text { such that } \\
\left(\boldsymbol{\nabla} w^{h}, \boldsymbol{\nabla} \varphi^{h}\right)=\left(\boldsymbol{\psi}_{0}^{h}+\boldsymbol{\psi}_{\mathcal{H}}^{h}, \nabla \times \varphi^{h}\right),
\end{array}\right. \\
& \left\{\begin{array}{l}
\text { Find } \boldsymbol{u}^{h} \in\left[S^{h, k}\right]^{2} \text { such that } \\
\left(\boldsymbol{u}^{h}, \boldsymbol{\varphi}^{h}\right)=\left(\boldsymbol{\nabla} \times w^{h}+\nabla q^{h}, \boldsymbol{\varphi}^{h}\right),
\end{array}\right.
\end{aligned}
$$

Subproblems $(9.7,9.8,9.9 . \mathrm{i}, 9.10)$ and (9.11) can be easily solved in an uncoupled way. The coupled subproblem (9.9.ii) can be solved either by the direct decomposition method of Glowinski and Pironneau (see e.g. $[1,15,16]$ ) or by one of its iterative variants, such as, for instance, the conjugate gradient method (see $[7,10]$ ).

Remark 9.1. In the particular case of $\Omega$ rectangular domain, the Quartapelle-Muzzio problem (8.3) can be written naturally as a system of two independent scalar Poisson equations each supplemented with mixed Dirichlet-Neumann conditions. In this case an uncoupled solution of problem (7.1) is obtained directly.

\section{Convergence Analysis}

Let the triangulation $\mathcal{T}^{h}$ belong to a quasi-uniform family (see e.g. [4]) and denote below by $\|\cdot\|_{s}$ and $|\cdot|_{s}$ the standard norm and seminorm of the Sobolev space $H^{s}(\Omega), s>0$. We denote by $C$ a generic constant independent of $h$.

Estimating $\phi-\phi^{h}$. Since $\phi^{h}$ is a conforming $P_{k}$ finite element approximation of $\phi$, this estimates is classical.

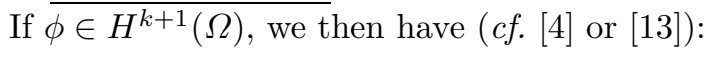

$$
\left\|\phi-\phi^{h}\right\|_{0}+h\left|\phi-\phi^{h}\right|_{1} \leq C h^{k+1}|\phi|_{k+1} .
$$

$\underline{\text { Estimating } q-q^{h}}$. From (8.10) and (9.8), we can get

$$
\left(\nabla\left(q-q^{h}\right), \nabla \varphi^{h}\right)=-\left(\phi-\phi^{h}, \varphi^{h}\right), \quad \forall \varphi^{h} \in S_{n}^{h, j},
$$

thus,

$$
\begin{aligned}
\left\|\nabla\left(q^{h}-\varphi^{h}\right)\right\|_{0}^{2} & =\left(\boldsymbol{\nabla}\left(q-\varphi^{h}\right), \boldsymbol{\nabla}\left(q^{h}-\varphi^{h}\right)\right)-\left(\boldsymbol{\nabla}\left(q-q^{h}\right), \boldsymbol{\nabla}\left(q^{h}-\varphi^{h}\right)\right) \\
& =\left(\boldsymbol{\nabla}\left(q-\varphi^{h}\right), \boldsymbol{\nabla}\left(q^{h}-\varphi^{h}\right)\right)+\left(\phi-\phi^{h}, q^{h}-\varphi^{h}\right) \\
& \leq C\left\{\left\|\boldsymbol{\nabla}\left(q-\varphi^{h}\right)\right\|_{0}+\left\|\phi-\phi^{h}\right\|_{0}\right\}\left\|\nabla\left(q^{h}-\varphi^{h}\right)\right\|_{0}, \quad \forall \varphi^{h} \in S_{0}^{h, j},
\end{aligned}
$$

if $q \in H^{j+1}(\Omega)$ and $\phi \in H^{k+1}(\Omega)$. Hence, by the classical interpolation results and the estimate (10.1), we have, for $j=k$ or $k+1$

$$
\begin{aligned}
\left\|\nabla\left(q-q^{h}\right)\right\|_{0} & \leq \inf _{\varphi^{h} \in S_{0}^{h, j}}\left\{\left\|\boldsymbol{\nabla}\left(q-\varphi^{h}\right)\right\|_{0}+\left\|\boldsymbol{\nabla}\left(q^{h}-\varphi^{h}\right)\right\|_{0}\right\} \\
& \leq C\left\{\inf _{\varphi^{h} \in S_{0}^{h, j}}\left\|\boldsymbol{\nabla}\left(q-\varphi^{h}\right)\right\|_{0}+\left\|\phi-\phi^{h}\right\|_{0}\right\} \\
& \leq C h^{j}\left\{|q|_{j+1}+|\phi|_{k+1}\right\} .
\end{aligned}
$$


$\underline{\text { Estimating } \boldsymbol{\psi}_{0}-\boldsymbol{\psi}_{0}^{h}}$. By (8.16.i) and (9.9.ii), we have

$$
\begin{aligned}
\left|\boldsymbol{\psi}_{0}^{h}-\boldsymbol{\varphi}_{0}^{h}\right|_{1}^{2} & =\left(\boldsymbol{\psi}_{0}-\boldsymbol{\varphi}_{0}^{h}, \boldsymbol{\psi}_{0}^{h}-\boldsymbol{\varphi}_{0}^{h}\right)_{1}-\left(\boldsymbol{\psi}_{0}-\boldsymbol{\psi}_{0}^{h}, \boldsymbol{\psi}_{0}^{h}-\boldsymbol{\varphi}_{0}^{h}\right)_{1} \\
& =\left(\boldsymbol{\psi}_{0}-\boldsymbol{\varphi}_{0}^{h}, \boldsymbol{\psi}_{0}^{h}-\boldsymbol{\varphi}_{0}^{h}\right)_{1}-\left(\boldsymbol{\nabla}\left(\phi-\phi^{h}\right), \boldsymbol{\psi}_{0}^{h}-\boldsymbol{\varphi}_{0}^{h}\right) \\
& =\left(\boldsymbol{\psi}_{0}-\boldsymbol{\varphi}_{0}^{h}, \boldsymbol{\psi}_{0}^{h}-\boldsymbol{\varphi}_{0}^{h}\right)_{1}+\left(\phi-\phi^{h}, \boldsymbol{\nabla} \cdot\left(\boldsymbol{\psi}_{0}^{h}-\boldsymbol{\varphi}_{0}^{h}\right)\right) \\
& \leq C\left\{\left|\boldsymbol{\psi}_{0}-\boldsymbol{\varphi}_{0}^{h}\right|_{1}+\left\|\phi-\phi^{h}\right\|_{0}\right\}\left|\boldsymbol{\psi}_{0}^{h}-\boldsymbol{\varphi}_{0}^{h}\right|_{1}, \quad \forall \boldsymbol{\varphi}_{0}^{h} \in\left[S_{0}^{h, j}\right]^{2} .
\end{aligned}
$$

Similarly to the above deduction, one can get

$$
\left|\boldsymbol{\psi}_{0}-\boldsymbol{\psi}_{0}^{h}\right|_{1} \leq C h^{j}\left\{\left|\boldsymbol{\psi}_{0}\right|_{j+1}+|\phi|_{k+1}\right\}
$$

if $\boldsymbol{\psi}_{0} \in \boldsymbol{H}^{j+1}(\Omega)$ and $\phi \in H^{k+1}(\Omega)$.

Estimating $\boldsymbol{\psi}_{\mathcal{H}}-\boldsymbol{\psi}_{\mathcal{H}}^{h}$. This is a little harder. First, according to Strang's Lemma (cf. [4]), we have:

$$
\left|\boldsymbol{\psi}_{\mathcal{H}}-\boldsymbol{\psi}_{\mathcal{H}}^{h}\right|_{1} \leq C\left\{\inf _{\boldsymbol{\varphi}_{\mathcal{H}}^{h} \in \mathcal{H}_{N}^{h, j}}\left|\boldsymbol{\psi}_{\mathcal{H}}-\boldsymbol{\varphi}_{\mathcal{H}}^{h}\right|_{1}+\sup _{\boldsymbol{\varphi}_{\mathcal{H}}^{h} \in \mathcal{H}_{N}^{h, j} \backslash\{\mathbf{0}\}} \frac{\left|\left(\boldsymbol{\psi}_{\mathcal{H}}, \boldsymbol{\varphi}_{\mathcal{H}}^{h}\right)_{1}-L_{h}\left(\boldsymbol{\varphi}_{\mathcal{H}}^{h}\right)\right|}{\left|\boldsymbol{\varphi}_{\mathcal{H}}^{h}\right|_{1}}\right\}
$$

where

$$
L_{h}\left(\boldsymbol{\varphi}_{\mathcal{H}}^{h}\right)=-\left(\boldsymbol{\psi}_{0}^{h}, \boldsymbol{\varphi}_{\mathcal{H}}^{h}\right)_{1}+\left(\boldsymbol{f}+\nabla \phi^{h}, \boldsymbol{\varphi}_{\mathcal{H}}^{h}\right)
$$

To estimate the right-hand side of (10.4), we have

Lemma 10.1. If $\boldsymbol{\psi}_{\mathcal{H}} \in \mathcal{H}_{N}(\Omega) \bigcap \boldsymbol{H}^{j+1}(\Omega)(j=k$ or $k+1)$, then there exists a constant $C$ independent of $h$ such that

$$
\inf _{\boldsymbol{\varphi}_{\mathcal{H}}^{h} \in \mathcal{H}_{N}^{h, j}}\left\{\left\|\boldsymbol{\psi}_{\mathcal{H}}-\boldsymbol{\varphi}_{\mathcal{H}}^{h}\right\|_{0}+h\left|\boldsymbol{\psi}_{\mathcal{H}}-\boldsymbol{\varphi}_{\mathcal{H}}^{h}\right|_{1}\right\} \leq C h^{j+1}\left|\boldsymbol{\psi}_{\mathcal{H}}\right|_{j+1}
$$

Proof. Let us take the standard elliptic projection of $\boldsymbol{\psi}_{\mathcal{H}}$ into the finite element space $\left[S^{h, j}\right]^{2}$ denoted by $\mathbf{P}_{1} \boldsymbol{\psi}_{\mathcal{H}}$, which is defined by:

$$
\left\{\begin{array}{l}
\left(\mathbf{P}_{1} \boldsymbol{\psi}_{\mathcal{H}}-\boldsymbol{\psi}_{\mathcal{H}}, \boldsymbol{\varphi}^{h}\right)_{1}=0, \quad \forall \boldsymbol{\varphi}^{h} \in\left[S_{0}^{h, j}\right]^{2} \\
\mathbf{P}_{1} \boldsymbol{\psi}_{\mathcal{H} \mid \Gamma}=\mathcal{I}_{j} \boldsymbol{\psi}_{\mathcal{H}} \mid \Gamma
\end{array}\right.
$$

By classical results (see [4]), we have

$$
\left\|\boldsymbol{\psi}_{\mathcal{H}}-\mathbf{P}_{1} \boldsymbol{\psi}_{\mathcal{H}}\right\|_{0}+h\left|\boldsymbol{\psi}_{\mathcal{H}}-\mathbf{P}_{1} \boldsymbol{\psi}_{\mathcal{H}}\right|_{1} \leq C h^{j+1}\left|\boldsymbol{\psi}_{\mathcal{H}}\right|_{j+1}
$$

On the other hand, since $\boldsymbol{\psi}_{\mathcal{H}} \in \mathcal{H}_{N}(\Omega)$, by the definition of $\mathbf{P}_{1} \boldsymbol{\psi}_{\mathcal{H}}$, it is easy to show that $\mathbf{P}_{1} \boldsymbol{\psi}_{\mathcal{H}} \in \boldsymbol{S}_{N}^{h, j}$ and

$$
\left(\mathbf{P}_{1} \boldsymbol{\psi}_{\mathcal{H}}, \varphi^{h}\right)_{1}=\left(\boldsymbol{\psi}_{\mathcal{H}}, \varphi^{h}\right)_{1}=-\left(\nabla^{2} \boldsymbol{\psi}_{\mathcal{H}}, \varphi^{h}\right)=0, \quad \forall \boldsymbol{\varphi}^{h} \in\left[S_{0}^{h, j}\right]^{2}
$$

This means that $\mathbf{P}_{1} \boldsymbol{\psi}_{\mathcal{H}} \in \mathcal{H}_{N}^{h, j}$. Then, let $\boldsymbol{\varphi}_{\mathcal{H}}^{h}=\mathbf{P}_{1} \boldsymbol{\psi}_{\mathcal{H}}$ to complete the proof.

Lemma 10.2. If $\boldsymbol{\psi}_{0} \in \boldsymbol{H}^{j+1}(\Omega)$ and $\phi \in H^{k+1}(\Omega)$, then, for $j=k$ or $k+1$

$$
\sup _{\boldsymbol{\varphi}_{\mathcal{H}}^{h} \in \mathcal{H}_{N}^{h, j} \backslash\{\mathbf{0}\}} \frac{\left|\left(\boldsymbol{\psi}_{\mathcal{H}}, \boldsymbol{\varphi}_{\mathcal{H}}^{h}\right)_{1}-L_{h}\left(\boldsymbol{\varphi}_{\mathcal{H}}^{h}\right)\right|}{\left|\boldsymbol{\varphi}_{\mathcal{H}}^{h}\right|_{1}} \leq C h^{j}\left\{\left|\boldsymbol{\psi}_{0}\right|_{j+1}+|\phi|_{k+1}\right\} .
$$


Proof. Since $\boldsymbol{\varphi}_{\mathcal{H}}^{h} \in \mathcal{H}_{N}^{h, j} \subset \boldsymbol{X}_{N}(\Omega)$, owing to (8.11) and (8.14) we have

$$
\left(\boldsymbol{\psi}_{\mathcal{H}}, \varphi_{\mathcal{H}}^{h}\right)_{1}=-\left(\boldsymbol{\psi}_{0}, \varphi_{\mathcal{H}}^{h}\right)_{1}+\left(\boldsymbol{f}+\boldsymbol{\nabla} \phi, \varphi_{\mathcal{H}}^{h}\right), \quad \forall \varphi_{\mathcal{H}}^{h} \in \mathcal{H}_{N}^{h, j}
$$

Thus, for any $\varphi_{\mathcal{H}}^{h} \in \mathcal{H}_{N}^{h, j}$, we have

$$
\begin{aligned}
\left|\left(\boldsymbol{\psi}_{\mathcal{H}}, \boldsymbol{\varphi}_{\mathcal{H}}^{h}\right)_{1}-L_{h}\left(\boldsymbol{\varphi}_{\mathcal{H}}^{h}\right)\right| & \leq\left|\left(\boldsymbol{\psi}_{0}-\boldsymbol{\psi}_{0}^{h}, \boldsymbol{\varphi}_{\mathcal{H}}^{h}\right)_{1}\right|+\left|\left(\boldsymbol{\nabla}\left(\phi-\phi^{h}\right), \boldsymbol{\varphi}_{\mathcal{H}}^{h}\right)\right| \\
& \leq\left|\left(\boldsymbol{\psi}_{0}-\boldsymbol{\psi}_{0}^{h}, \boldsymbol{\varphi}_{\mathcal{H}}^{h}\right)_{1}\right|+\left|\left(\phi-\phi^{h}, \boldsymbol{\nabla} \cdot \boldsymbol{\varphi}_{\mathcal{H}}^{h}\right)\right| \\
& \leq\left\{\left|\boldsymbol{\psi}_{0}-\boldsymbol{\psi}_{0}^{h}\right|_{1}+\left\|\phi-\phi^{h}\right\|_{0}\right\}\left|\boldsymbol{\varphi}_{\mathcal{H}}^{h}\right|_{1} .
\end{aligned}
$$

Therefore, by (10.1) and (10.3), we know that the estimate (10.9) holds.

By (10.4), (10.6) and (10.9), we obtain

$$
\left|\boldsymbol{\psi}_{\mathcal{H}}-\boldsymbol{\psi}_{\mathcal{H}}^{h}\right|_{1} \leq C h^{j}\left\{\left|\boldsymbol{\psi}_{0}\right|_{j+1}+\left|\boldsymbol{\psi}_{\mathcal{H}}\right|_{j+1}+|\phi|_{k+1}\right\},
$$

$j=k$ or $k+1$.

$\underline{\text { Estimating } w-w^{h}}$. Similarly to estimate $q-q^{h}$, by (8.12) and (9.10), we can get

$$
\begin{aligned}
\left|w-w^{h}\right|_{1} & \leq C\left\{\inf _{\varphi^{h} \in S_{0}^{h, j}}\left|w-\varphi^{h}\right|_{1}+\left\|\boldsymbol{\psi}_{0}-\boldsymbol{\psi}_{0}^{h}\right\|_{0}+\left\|\boldsymbol{\psi}_{\mathcal{H}}-\boldsymbol{\psi}_{\mathcal{H}}^{h}\right\|_{0}\right\} \\
& \leq C h^{j}\left\{|w|_{j+1}+\left|\boldsymbol{\psi}_{0}\right|_{j+1}+\left|\boldsymbol{\psi}_{\mathcal{H}}\right|_{j+1}+|\phi|_{k+1}\right\}
\end{aligned}
$$

if $w \in H^{j+1}(\Omega), \boldsymbol{\psi}_{0}, \boldsymbol{\psi}_{\mathcal{H}} \in \boldsymbol{H}^{j+1}(\Omega)$ and $\phi \in H^{k+1}(\Omega), j=k$ or $k+1$.

$\underline{\text { Estimating } \boldsymbol{u}-\boldsymbol{u}^{h}}$. By (8.13) and (9.11), notice that (10.2) and (10.11), we have

$$
\begin{aligned}
\left\|\boldsymbol{u}-\boldsymbol{u}^{h}\right\|_{0} & \leq C\left\{\inf _{\boldsymbol{\varphi}^{h} \in\left[S_{0}^{h, k}\right]^{2}}\left\|\boldsymbol{u}-\boldsymbol{\varphi}^{h}\right\|_{0}+\left\|\boldsymbol{\nabla}\left(q-q^{h}\right)\right\|_{0}+\left|w-w^{h}\right|_{1}\right\} \\
& \leq C h^{j}\left\{|q|_{j+1}+|w|_{j+1}+\left|\boldsymbol{\psi}_{0}\right|_{j+1}+\left|\boldsymbol{\psi}_{\mathcal{H}}\right|_{j+1}+|\boldsymbol{u}|_{k+1}+|\phi|_{k+1}\right\},
\end{aligned}
$$

if $q, w \in H^{j+1}(\Omega), \boldsymbol{\psi}_{0}, \boldsymbol{\psi}_{\mathcal{H}} \in \boldsymbol{H}^{j+1}(\Omega), \boldsymbol{u} \in \boldsymbol{H}^{k+1}(\Omega)$ and $\phi \in H^{k+1}(\Omega), j=k$ or $k+1$.

Summarizing the analysis above, we obtain:

Theorem 10.1. Assume that $\phi, q, \boldsymbol{\psi}_{0}, \boldsymbol{\psi}_{\mathcal{H}}, w$ and $\boldsymbol{u}$, the solutions of problems (8.9), (8.10), (8.16.i), (8.16.ii), (8.12) and (8.13), belong to $H^{k+1}(\Omega), H^{j+1}(\Omega), \boldsymbol{H}^{j+1}(\Omega), \boldsymbol{H}^{j+1}(\Omega), H^{j+1}(\Omega)$ and $\boldsymbol{H}^{k+1}(\Omega)$, for $j=k$ or $k+1$, respectively. $\phi^{h}, q^{h}, \boldsymbol{\psi}_{0}^{h}, \boldsymbol{\psi}_{\mathcal{H}}^{h}, w^{h}$ and $\boldsymbol{u}^{h}$ are the finite element solutions of (9.7), (9.8), (9.9.i), (9.9.ii), (9.10) and (9.11), respectively. Then there exists a constant $C$ independent of $h$ such that the following error estimates hold:

$$
\left\|\phi-\phi^{h}\right\|_{0}+h\left|\phi-\phi^{h}\right|_{1} \leq C h^{k+1}|\phi|_{k+1},
$$




$$
\begin{gathered}
\left|q-q^{h}\right|_{1} \leq C h^{j}\left\{|q|_{j+1}+|\phi|_{k+1}\right\}, \\
\left|\boldsymbol{\psi}_{0}-\boldsymbol{\psi}_{0}^{h}\right|_{1} \leq C h^{j}\left\{\left|\boldsymbol{\psi}_{0}\right|_{j+1}+|\phi|_{k+1}\right\} \\
\left|\boldsymbol{\psi}_{\mathcal{H}}-\boldsymbol{\psi}_{\mathcal{H}}^{h}\right|_{1} \leq C h^{j}\left\{\left|\boldsymbol{\psi}_{0}\right|_{j+1}+\left|\boldsymbol{\psi}_{\mathcal{H}}\right|_{j+1}+|\phi|_{k+1}\right\} \\
\left|w-w^{h}\right|_{1} \leq C h^{j}\left\{|w|_{j+1}+\left|\boldsymbol{\psi}_{0}\right|_{j+1}+\left|\boldsymbol{\psi}_{\mathcal{H}}\right|_{j+1}+|\phi|_{k+1}\right\} \\
\left\|\boldsymbol{u}-\boldsymbol{u}^{h}\right\|_{0} \leq C h^{j}\left\{|q|_{j+1}+|w|_{j+1}+\left|\boldsymbol{\psi}_{0}\right|_{j+1}+\left|\boldsymbol{\psi}_{\mathcal{H}}\right|_{j+1}+|\boldsymbol{u}|_{k+1}+|\phi|_{k+1}\right\}
\end{gathered}
$$

Remark 10.1. By Theorem 10.1, if $j=k+1$, then we get an optimal convergence result for approximating $\boldsymbol{u}$ with order of $\mathcal{O}\left(h^{k+1}\right)$. If we choose $j=k$, then the convergence result only can reach $\mathcal{O}\left(h^{k}\right)$. In the special case $k=1$, the linear finite elements can be applied to all of approximations.

\section{Numerical RESUlts}

The uncoupled solution method described in the previous sections has been implemented by considering a finite element approximation by linear interpolation of the unknown $\boldsymbol{u}$ as well as of all the intermediate variables. The numerical error of the computed solutions has been characterized by the relative error in the $L^{2}$ norm, namely

$$
\left\|v^{\mathrm{ex}}-v^{\mathrm{c}}\right\|_{0} /\left\|v^{\mathrm{ex}}\right\|_{0}
$$

where $v^{\text {ex }}$ is the interpolate of the exact analytical solution of a scalar problem and $v^{\mathrm{c}}$ the computed solution. Similarly, the relative $H^{1}$ error is defined as follows:

$$
\left\|v^{\mathrm{ex}}-v^{\mathrm{c}}\right\|_{1} /\left\|v^{\mathrm{ex}}\right\|_{1}
$$

where $\|\cdot\|_{1}=\left\{\|\cdot\|_{0}^{2}+\|\nabla \cdot\|_{0}^{2}\right\}^{1 / 2}$

We have considered a test problem on the square $[-1,1]^{2}$ with an exact solution $\boldsymbol{u}=\boldsymbol{\nabla} \times w+\nabla q$ generated from the functions $w=\left(1-x^{2}\right)\left(1-y^{2}\right) y$ and $q=x e^{y}$. This problem has been solved on a sequence of uniform meshes of $2 \times 10^{2}, 2 \times 20^{2}, 2 \times 40^{2}$ and $2 \times 80^{2}$ equal triangles. Notice that the values of the datum $b$ for the derivative condition has been evaluated from the analytical solution at the Gaussian quadrature points of the surface elements, instead of being computed from the linear interpolate constructed with the values of $b$ at the surface nodes. The relative $L^{2}$ and $H^{1}$ errors and the ratios between the errors on two successively finer grids 
are reported in the Tables 1 and 2 , respectively.

TABLE 1. Relative $L^{2}$ error and error ratios.

\begin{tabular}{||c||c||c|c||c|c||c|c||}
\hline \hline & $N=10$ & \multicolumn{2}{c||}{$N=20$} & \multicolumn{2}{c||}{$N=40$} & \multicolumn{2}{c||}{$N=80$} \\
\hline & r.e. & r.e. & e.r. & r.e. & e.r. & r.e. & e.r. \\
\hline$\phi$ & 0.0014194 & 0.0003885 & 3.60 & 0.0000991 & 3.92 & 0.0000249 & 3.98 \\
$q$ & 0.0113205 & 0.0027763 & 4.08 & 0.0006890 & 4.02 & 0.0001718 & 4.01 \\
$w$ & 0.0446008 & 0.0114740 & 3.89 & 0.0028878 & 3.97 & 0.0007230 & 3.99 \\
$\boldsymbol{u}$ & 0.0586064 & 0.0196050 & 2.99 & 0.0065578 & 2.99 & 0.0022280 & 2.94 \\
\hline \hline
\end{tabular}

TABLE 2. Relative $H^{1}$ error and error ratios.

\begin{tabular}{||c||c||c|c||c|c||c|c||}
\hline \hline \multicolumn{1}{||c||}{} & \multicolumn{1}{c||}{$N=10$} & \multicolumn{2}{c||}{$N=20$} & \multicolumn{2}{c||}{$N=40$} & \multicolumn{2}{c||}{$N=80$} \\
\hline & r.e. & r.e. & e.r. & r.e. & e.r. & r.e. & e.r. \\
\hline$\phi$ & 0.0021799 & 0.0006809 & 3.20 & 0.0001917 & 3.55 & 0.0000507 & 3.78 \\
$q$ & 0.0212837 & 0.0059481 & 3.57 & 0.0016242 & 3.66 & 0.0004372 & 3.71 \\
$w$ & 0.0454291 & 0.0116969 & 3.88 & 0.0029452 & 3.97 & 0.0007375 & 3.99 \\
\hline \hline
\end{tabular}

\section{REFERENCES}

[1] Y. Achdou, R. Glowinski and O. Pironneau, Tuning the mesh of a mixed method for the stream function-vorticity formulation of the Navier-Stokes equations. Numer. Math. 63 (1992) 145-163.

[2] I. Babuška, The finite element method with Lagrange multipliers. Numer. Math. 20 (1973) $179-192$.

[3] C. Bernardi, Méthodes d'éléments finis mixtes pour les équations de Navier-Stokes. Thèse de $3^{\mathrm{e}}$ Cycle, Université de Paris VI, France (1979).

[4] P.G. Ciarlet, The Finite Element Method for Elliptic Problems. North-Holland, Amsterdam (1978).

[5] F. El Dabaghi and O. Pironneau, Stream vectors in three dimensional aerodynamics. Numer. Math. 48 (1986) $561-589$.

[6] V. Girault and P.-A. Raviart, Finite Element Methods for Navier-Stokes Equations. Springer-Verlag, Berlin (1986).

[7] R. Glowinski and O. Pironneau, Numerical methods for the first biharmonic equation and for the two-dimensional Stokes problem. SIAM Rev. 21 (1979) 167-212.

[8] P. Neittaanmaki and M. Krizek, in Efficient Solution of Elliptic Systems, Finite element approximation for a div-rot system with mixed boundary conditions in non-smooth plane domain. Notes in Numerical Fluid Mechanics, Vol. 10, W. Hachbush Ed., Vieweg Publishing, Wiesbaden, Germany (1984); see also Appl. Math. 29 (1984) 272-285.

[9] L. Quartapelle, Numerical Solution of the Incompressible Navier-Stokes Equations. Birkhäuser, Basel (1993).

[10] L. Quartapelle and A. Muzzio, Decoupled solution of vector Poisson equations with boundary condition coupling, in Computional Fluid Dynamics, G. de Vahl Davis and C. Fletcher Eds., Elsevier Science Publishers B.V., North-Holland (1988) 609-619.

[11] L. Quartapelle, V. Ruas and J. Zhu, Uncoupled solution of the three-dimensional vorticity-velocity equations. ZAMP 49 (1998) $384-400$.

[12] V. Ruas, L. Quartapelle and J. Zhu, A symmetrized velocity-vorticity formulation of the three-dimensional Stokes system. C.R. Acad. Sci. Paris Sér. IIb 323 (1996) 819-824.

[13] G. Strang and G.J. Fix, An Analysis of the Finite Element Method, Prentice-Hall, Englewood Cliffs, New York (1973).

[14] J. Zhu, A.F.D. Loula and L. Quartapelle, A vector Poisson problem with coupling boundary conditions in a Lipschitz 2D domain, Research Report, Laboratório Nacional de Computação Científica, CNPq, N 30 (1997).

[15] J. Zhu, A. F. D. Loula and L. Quartapelle, Finite element solution of vector Poisson equation with a coupling boundary condition. Numer. Methods Partial Differential Eq. 16 (2000).

[16] J. Zhu, L. Quartapelle and A.F.D. Loula, Uncoupled variational formulation of a vector Poisson problem. C.R. Acad. Sci. Paris Sér. I 323 (1996) 971-976. 